\title{
Anisotropy in the sky distributions of the short and intermediate gamma-ray bursts: Breakdown of the cosmological principle?
}

\author{
A. Mészáros*, L.G. Balázs ${ }^{\dagger}$, Z. Bagoly** and P. Veres** \\ ${ }^{*}$ Charles University, Prague, Czech Republic \\ ${ }^{\dagger}$ Konkoly Observatory, Budapest, Hungary \\ ${ }^{* *}$ Eötvös University, Budapest, Hungary
}

\begin{abstract}
After the discovery of the anisotropy in the sky-distribution of intermediate gamma-ray bursts recently also the distribution of the short gamma-ray bursts is proven to be anisotropic. The impact of these behaviors on the validity of the cosmological principle is shortly discussed.
\end{abstract}

Keywords: $\gamma$-ray sources; $\gamma$-ray bursts

PACS: $98.70 . \mathrm{Rz}$

\section{INTRODUCTION}

The cosmological principle requires that the Universe be spatially homogeneous and isotropic in average on scales larger than the size of any structure [1]. Observations show that the greatest structures (filaments, voids, superclusters, ...) have sizes around $400 \mathrm{Mpc}$ [2]. Hence, at redshifts $z<0.1$ the matter distribution in the Universe is anisotropic and inhomogeneous. There are further supports (both observational $[3,4,5,6]$ and theoretical $[7,8]$ ones) that such structures exist also at redshift $z<1$. If the structures with sizes $\simeq 400 \mathrm{Mpc}$ are not distributed isotropically at $z<1$, then some structures should exist even with sizes $\simeq(0.4-3.3) \mathrm{Gpc}$. In other words, if this is the case, then the scale - where the averaging should be done - should be at least of order $\sim 1 G p c$. In this case the fulfilement of the cosmological principle would be in doubt.

The angular distribution of the gamma-ray bursts (GRBs) allows to test this principle too, because - if this principle holds - GRBs should be distributed isotropically on the sky, if they are dominantly at $z>0.1$. For this test it is convenient that GRBs are well seen in the gamma-band also in the Galactic plane.

At the last years the authors provided several different tests probing the intrinsic isotropy in the angular skydistribution of GRBs collected in BATSE Catalog (for the survey and for further details see [9]). Shortly summarizing the results of these studies one may conclude: A. The long subgroup $\left(T_{90}>10 s\right)$ seems to be distributed isotropically; B. The intermediate subgroup $\left(2 s \leq T_{90} \leq 10 s\right)$ is distributed anisotropically on the $\simeq(96-97) \%$ significance level; C. For the short subgroup $\left(2 s>T_{90}\right)$ the assumption of isotropy is rejected only on the $92 \%$ significance level; D. The long and the short subclasses, respectively, are distributed differently on the $99.3 \%$ significance level. (About the definition of subclasses and other relevant topics see, e.g., $[10,11,12,13,14,15] ; T_{90}$ is the duration of a GRB, during which time the $90 \%$ of the radiated energy is received.)

\section{DESCRIPTION OF THE NEWEST TESTS}

Because mainly the situation concerning the short GRBs was unclear, we provided a new more powerful testing of the isotropy on the BATSE data. We used three methods. (The detailed description of these three methods with references is given in [9].)

The first one is the method called Voronoi tesselation (VT). The Voronoi diagram - also known as Dirichlet tesselation or Thiessen polygons - is a fundamental structure in computational geometry. Generally, this diagram provides a partition of a point pattern ("point field", also "point process") according to its spatial structure, which can be used for analyzing the underlying point process. The points on sphere may be distributed completely randomly or non-randomly; the non-random distribution may have different characters (clustering, filaments, etc.). 
The second method is called Minimal spanning tree (MST). Contrary to VT, this method considers the distances (edges) among the points (vertices). Clearly, there are $N(N-1) / 2$ distances among $N$ points. A spanning tree is a system of lines connecting all the points without any loops. The MST is a system of connecting lines, where the sum of the lengths is minimal among all the possible connections between the points. In our study the spherical version of MSF was used. The $N-1$ separate connecting lines (edges) together define the minimal spanning tree. The statistics of the lengths and the $\alpha_{M S T}$ angles between the edges at the vertices can be used for testing the randomness of the point pattern.

The third method uses the Multifractal spectrum (MS). The idea here is the following: Let denote $P(\varepsilon)$ the probability for finding a point in an area of $\varepsilon$ radius. If $P(\varepsilon)$ scales as $\varepsilon^{\alpha}$ (i.e. $P(\varepsilon) \propto \varepsilon^{\alpha}$ ), then $\alpha$ is called the local fractal dimension (e.g. $\alpha=2$ for a completely random process on the plane). In the case of a monofractal $\alpha$ is independent on the position. A multifractal (MFR) on a point process can be defined as unification of the subsets of different (fractal) dimensions. One usually denotes with $f(\alpha)$ the fractal dimension of the subset of points at which the local fractal dimension is in the interval of $\alpha, \alpha+d \alpha$. The contribution of these subsets to the whole pattern is not necessarily equally weighted, practically it depends on the relative abundances of subsets. The $f(\alpha)$ functional relationship between the fractal dimension of subsets and the corresponding local fractal dimension is called the MFR or Hausdorff spectrum.

The randomness of the point field on the sphere can be tested with respect to different criteria. Since different non-random behaviors are sensitive for different types of criteria of non-randomness, it is not necessary that all possible tests using different measures reject the assumption of randomness. We defined several test-variables which are sensitive to different stochastic properties of the underlying point pattern. For VT any of the four quantities characterizing the Voronoi cell - i.e. the area, the perimeter, the number of vertices, and the inner angles - can be used as test-variables or even some of their combinations, too. We used three quantities obtained for MST: variance of the MST edge-length $\left(\sigma\left(L_{M S T}\right)\right)$; mean MST edge-length $\left(L_{M S T}\right)$; and the mean angle between edges $\left(\alpha_{M S T}\right)$. For the multifractal spectrum the only used variable is the $f(\alpha)$ multifractal spectrum, which is a sensitive tool for testing the non-randomness of a point pattern. Together we had 13 variables, not being independent. The significance levels were calculated by Monte Carlo simulations.

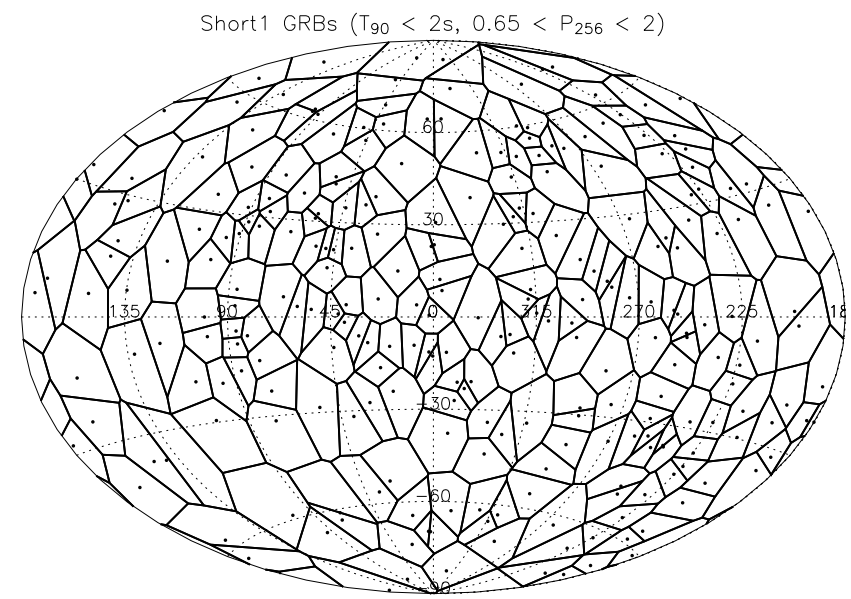

\section{THE SAMPLES}

We divided the BATSE's GRBs into three groups: short $\left(T_{90}<2 s\right)$, intermediate $\left(2 s \leq T_{90} \leq 10 s\right)$ and long $\left(T_{90}>10 s\right)$. To avoid the problems with the changing detection threshold we omitted the GRBs having a peak flux $P_{256} \leq$ 0.65 photons $\mathrm{cm}^{-2} \mathrm{~s}^{-1}$. The bursts may emerge at very different distances in the line of sight and it may happen that the stochastic structure of the angular distribution depends on it. Therefore, we also made tests on the bursts with $P_{256}<2$ photons $\mathrm{cm}^{-2} \mathrm{~s}^{-1}$ in the short and long population, separately. All this means that we studied five samples: "Short1" with $T_{90}<2 \mathrm{~s}$ and $0.65<P_{256}<2$ containing $261 \mathrm{GRBs}$; "Short2" with $T_{90}<2 \mathrm{~s}$ and $0.65<P_{256}$ containing 406 GRBs; "Intermediate" with $2 s \leq T_{90} \leq 10 s$ and $0.65<P_{256}$ containing 253 GRBs; "Long1" with $T_{90}>2 s$ and $0.65<P_{256}<2$ containing $676 \mathrm{GRBs}$; and "Long2" with $T_{90}>10 \mathrm{~s}$ and $0.65<P_{256}$ containing 966 GRBs. VT of the Short1 sample in Galactical coordinates is shown on the Figure. 


\section{THE RESULTS}

Both the Short1 and the Short2 samples deviate significantly on the $99.90 \%$ and $99.98 \%$ significance levels from the full randomness. Also the Intermediate sample gave a significant deviation $(\mathbf{9 8 . 5 1 \%})$ from the full randomness in accordance with the earlier study [16]. The long samples remained random.

\section{DISCUSSION AND CONCLUSION}

The subgroups of GRBs, detected by BATSE, show different sky distribution: Both the short and intermediate ones are distributed anisotropically on a high significance level; the long ones seem to be distributed isotropically. The situation concerning the intermediate ones is unclear (Is it a physically different subgroup or not?), but there is no doubt that the short and long ones are different phenomena. Because the short ones are distributed anisotropically (in addition - on large angular scales [17]), up to the redshifts - where the short ones dominate - the cosmological principle hardly can be fulfilled. The directly measured redshifts of the short GRBs - detected by Swift [18] - suggest that just this happens up to $z<1$ (see Table 1 of [19]). It is not sure that the short GRBs detected by BATSE, and the short GRBs detected by Swift together with measured redshifts are at the same redshift range. But, even keeping in mind this eventuality, it is well possible that the validity of the cosmological principle is in doubt at $z<1$. A support for this point of view was given by [9].

Note here that a similar sceptical points of view about the validity of the cosmological principle were claimed, too, from the studies of fully different topics [20, 21, 22].

\section{ACKNOWLEDGEMENTS}

This research was supported from OTKA grants T048870 and T077795, by a GAUK grant No.46307, by the Research Program MSM0021620860 of the Ministry of Education of the Czech Republic (A.M.), and by a Polányi grant KFKT2006-01-00012 (P.V.).

\section{REFERENCES}

1. P.J.E. Peebles, Principles of Physical Cosmology, Princeton Univ. Press (1993).

2. J.A. Peacock, Cosmological Physics, Cambridge Univ. Press (2000).

3. T.J. Broadhurst, et al., Nature 343, 726-728 (1990).

4. G. Paál, et al., ApSS 191, 107-124 (1992).

5. A. Holba, et al., ApSS 222, 65-83 (1994).

6. L. Rudnick, et al., ApJ 671, 40-44 (2007); Errata ApJ 678, 1531-1531 (2008).

7. I. Horváth, J Kor.Phys.Soc. 35, 629-632 (1999).

8. V. Springel, et al., Nature 440, 1137-1144 (2006).

9. R. Vavrek, et al., MNRAS 391, 1741-1748 (2008);

10. I. Horváth, ApJ 508, 757-759, (1998).

11. S. Mukherjee, et al., ApJ 508, 314-327, (1998).

12. I. Horváth, \& P. Mészáros, Bolyai Szemle 9, 71-88, (2000).

13. V.F. Litvin, et al,, Astronomy Letters 27, 416-420, (2001).

14. I. Horváth, $A \& A$ 392, 791-793, (2002).

15. I. Horváth, et al., $A \& A$ 498, L1-L4, (2008).

16. A. Mészáros, et al., ApJ 539, 98-101, (2000).

17. A. Mészáros, et al., $A \& A$ 234, 1-6, (2000).

18. http://heasarc.nasa.gov/docs/swift/swiftsc.html (Swift homepage).

19. D.A. Kann, et al., ApJ in press, (2008); astro-ph/0804.1959.

20. C.B. Collins \& S.W. Hawking, MNRAS 162, 307-320, (1973).

21. P. Birch, Nature 298, 451-454, (1982).

22. A. Kashlinsky, et al., ApJ 686, L49-L52 (2008). 